\title{
Serum Iron and Total Iron Binding Capacity in Burmese Patients with Leprosy
}

\author{
TIN SHWE \\ L.eprosy Ilospital, Il tuukkyant, Rangoon \\ THANE-TOE AND AUNG THAN BA TU \\ Department of Medical Research, Rangoon
}

\begin{abstract}
Serum iron and total iron binding capacity were estimated in the sera collected from 96 male leprosy patients (age 20-40 years) belonging to Leprosy Hospital, Htaukkyant. The sera from 22 healthy subjects were included in the study as controls. A low level of serum iron (below $50 \mu \mathrm{g} \%$ ) with normal iron binding capacity was recorded in 36 out of 60 patients $(60 \%$ with lepromatous leprosy, 14 out of 36 patients (39\%) with tuberculoid type and only 4 out of 22 controls $(18 \%)$. There was no difference in the prevalence of low serum iron and percentage saturation between those leprosy patients who had been on DDS and ferrous sulphate pills for at least 2 years and untreated new leprosy patients.

Bone marrow from 7 patients with anaemia and low serum iron, as well as the liver at autopsy from 3 male patients with advanced lepromatous leprosy and low socio-economic status showed normal iron content. Injection of parenteral iron (10 daily injections of Imferon) to 6 patients with anaemia and low serum iron also showed no increase in haemoglobin level.

Thus the anaemia in leprosy is not due to true lack of iron in the body (tissue iron deficiency). However, reduction in serum iron level is probably due in part to the toxic process of the disease upon the body.
\end{abstract}

\section{Introduction}

Anaemia is one of the common complications in patients with lepromatous leprosy. Low levels of serum iron with normal total iron binding capacity (TIBC) and transferrin in British patients with leprosy were found by Tin Shwe and his co-workers in 1972, but their study was based on a limited number of treated leprosy patients belonging to a developed country where the socio-economic status of the patients is entirely different from that of our country.

Burmese leprosy patients have been supplied with ferrous sulphate pills together with antileprosy drugs as a routine to prevent anaemia, but the usefulness of iron pills is not known. This study was carried out in order to establish the effect of ferrous sulphate pills on the iron status of leprosy patients. 


\section{Materials and Methods}

Estimation of serum iron and total iron binding capacity was carried out on the sera collected from a random sampling of 96 male leprosy patients (ages between 20-40 years) belonging to Htaukkyant Leprosy Hospital, Rangoon, Burma. Of these, 60 patients had lepromatous leprosy while the remaining 36 belonged to the non-lepromatous tuberculoid group. Half of the patients were untreated and newly diagnosed cases at the hospital while the remainder had been treated as in-patients of the hospital with antileprosy drugs (DDS 50-100 mg) and ferrous sulphate ( 5 grains or $325 \mathrm{mg}$ ) daily for not less than 2 years. All the patients were clinically free from renal and hepatic disorders. The blood samples were taken in the morning or afternoon and not in the evening.

The sera from 22 healthy males belonging to the same age group and who came to the hospital for skin infestations other than leprosy were included in the study as controls. The serum iron and total iron binding capacity were estimated by the method of Shade et al. (1954).

The haemosiderin content of the bone marrow from 7 patients with anaemia and low serum iron, and the liver non-Hb iron from 3 male autopsied patients with advanced lepromatous leprosy and low socio-economic status were also estimated.

Ten injections of parenteral iron $(2 \mathrm{ml} \mathrm{Imferon} \mathrm{each} \mathrm{containing} 100 \mathrm{mg}$ of iron as ferric hydroxide were given daily to 6 patients with anaemia and low serum iron level and their haemoglobin level was determined at weekly intervals for 4 to 6 weeks.

\section{Results}

Out of 60 patients with lepromatous leprosy, 36 patients $(60 \%)$ showed a reduction in iron level $(<50 \mu \mathrm{g} \%)$ with normal or reduced level of total iron binding capacity. There was no significant difference in the level of mean serum iron or TIBC between those patients who had not taken ferrous sulphate pills and those who had taken ferrous sulphate and DDS for not less than 2 years.

Out of 36 patients with tuberculoid leprosy, 14 patients (39\%) showed a reduction in serum iron level with normal or reduced total iron binding capacity. In this group also there was no significant difference in the level of serum iron or TIBC between those patients who had not taken ferrous sulphate pills and those who had taken ferrous sulphate and DDS for not less than 2 years.

Out of 22 controls, 4 subjects $(18.2 \%)$ showed reduction in serum iron level with normal level of total iron binding capacity. The details are given in Table 1.

The prevalence of patients with reduced serum iron level is significantly higher in patients with lepromatous leprosy when compared to those with tuberculoid leprosy and controls. The mean level of serum iron is also significantly reduced in patients with leprosy when compared to controls with the exception of the untreated lepromatous group. A similar significant difference in the level of total iron binding capacity between leprosy patients and controls is observed, with the exception of the untreated lepromatous group. However, the mean level of percentage saturation is not significantly reduced in patients with leprosy, the only exception being the treated lepromatous group.

Taking all untreated leprosy patients as a whole, 24 out of $50(48 \%)$ had serum iron level below $50 \mu \mathrm{g} \%$, while the treated group had 26 out of $46(56 \%)$ 
TABLE 1

Serum iron (SI) total hinding capacity (TIBC) and percentage saturation (\% sat.) of healthy controls and leprosy patients

\begin{tabular}{|c|c|c|c|c|c|c|c|c|}
\hline \multirow[b]{2}{*}{ Subjects } & \multirow[b]{2}{*}{ No. } & \multicolumn{3}{|c|}{ Mean \pm S.D. } & \multicolumn{4}{|c|}{ Number with } \\
\hline & & SI & TIBC & $\%$ Sat. & $\mathrm{SI}<$ & $\begin{array}{l}50 \mu \mathrm{g} \\
\%\end{array}$ & & $\begin{array}{l}\text { Sat. } \\
15\end{array}$ \\
\hline Control & 22 & $106 \pm 48$ & $338 \pm 71$ & $31 \pm 15$ & 4 & (18) & 5 & (23) \\
\hline \multicolumn{9}{|l|}{ Tuberculoid } \\
\hline Untreated & 19 & $74 \pm 47$ & $268 \pm 68$ & $28 \pm 15$ & 7 & (37) & 5 & $(26)$ \\
\hline Treated & 17 & $69 \pm 39$ & $275 \pm 61$ & $26 \pm 14$ & 7 & (41) & 6 & (35) \\
\hline 'Tuberculoid (all) & 36 & & & & 14 & (39) & 11 & (33) \\
\hline \multicolumn{9}{|l|}{ Lepromatous } \\
\hline Untreated & 31 & $74 \pm 56$ & $297 \pm 88$ & $25 \pm 15$ & 17 & $(55)$ & 12 & (26) \\
\hline Treated & 29 & $52 \pm 42$ & $266 \pm 85$ & $20 \pm 14$ & 19 & (66) & $\cdot 14$ & (20) \\
\hline Lepromatous (all) & 60 & & & & 36 & $(60)$ & 26 & $(20)$ \\
\hline Total leprosy & 96 & & & & 50 & $(52)$ & 37 & $(21)$ \\
\hline
\end{tabular}

Figures in parentheses represent percentage of respective total.

with low serum iron. Similarly 17 out of 50 (34\%) untreated leprosy patients and 20 out of $46(43 \%)$ treated patients had percentage saturation below 15 . Thus the prevalence of low serum iron and reduced percent saturation in leprosy patients is significantly higher when compared to those in the control group, i.e. 18 and $23 \%$ respectively).

Bone marrow taken from 7 patients with anaemia and low serum iron showed stainable iron (haemosiderin). The liver autopsy specimens taken from 3 male patients with advanced leprosy and low socio-economic status also showed normal iron content.

The 6 patients with anaemia and low serum iron who had been given parenteral iron ( 10 injections of Imferon) showed no increase in haemoglobin levels.

\section{Discussion}

Reduction in cell size and haemoglobin content are characteristic of the fully developed picture of iron deficiency anaemia in which the synthesis of haemoglobin is reduced. In the early evolution of this type of anaemia either of these morphological changes may be seen, but biochemical investigations reveal the underlying disorder that leads eventually to its production. The levels of plasma iron and its carrier protein transferrin most frequently measured as the total iron binding capacity (TIBC) of plasma and the percentage of saturation often provide a clue to the cause of defective haemoglobin synthesis (Turnbull, 1971).

In our investigation a random sample of male patients with ages between $20-40$ years was chosen for study because of the lesser risk of iron deficiency in males. 
Although serum iron and total iron binding capacity in patients with lepromatous leprosy did not differ significantly from those with non-lepromatous leprosy they were significantly lower than those of controls. This finding, however, is not in full agreement with the earlier study where Tin Shwe et al. (1972) using the spectrophotometric method found no reduction in total iron binding capacity, and serum transferrin level in patients with lepromatous leprosy, but the mean level of serum iron was significantly reduced in patients with lepromatous leprosy, though their method of serum iron estimation was different from that of the present study.

The lowered serum iron levels may be due to a reduction in iron supply, which again either may be due to a true lack of iron in the body (body iron deficiency) or to a failure in the delivery of iron from iron stores in bone marrow leading to what has been called "iron deficient erythropoisis". The true lack of iron in the body (body iron deficiency) would be due to an iron deficient diet, poor absorption of iron from the intestine, or excessive loss of iron following acute or chronic haemorrhage. The failure in delivery of iron from stores to bone marrow may occur if transporting protein transferrin is deficient, or if associated with chronic disorders (Cartwright, 1966; Cartwright and Lee, 1971).

In this present study the patients were free from acute and chronic haemorrhages, and defective iron absorption has not been reported in leprosy. The bone marrow taken from 7 patients with anaemia and leprosy, as well as the liver of 3 patients who had died of leprosy showed that there is no deficiency of iron in the stores. Therefore the reduction in serum iron level is probably due to impaired delivery of iron from the stores to the circulation as a result of the toxic process of the disease upon the body.

More than half of the patients who are on regular ferrous sulphate pills also showed reduced serum iron levels. Since those patients are provided with such pills daily to be taken in the presence of the nurses there is no possibility that they may not be taking the pills. Therefore the value of routine iron pills in leprosy remains to be judged from the cost effectiveness point of view. Instead, multivitamin and other measures may be needed to improve the general nutritional status of the patients.

\section{Acknowledgements}

We would like to thank Dr U. Kyaw Lwin, Deputy Director (Leprosy) Department of Health, Ministry of Health, Burma, Dr U. Eng I!oe, Medical Superintendent Leprosy Hospital, Htaukkyant, and the staff of Leprosy Hospital, Htaukkyant, Rangoon, for all their help.

\section{References}

Bainton, D. F. and Finch, C. A. (1964). The diagnosis of iron deficiency anaemia. Am. J. Med. $37,62-70$.

Cartwright, G. E. (1966). Seminars in Haematology 3, 351. Quoted in Turnbull, A. Br.J. Hosp. Med. (1971) 6, 573-580.

Cartwright, G. E. and Lee, G. R. (1971). The anaemia of chronic disorders (Annotation). Br. J. Haemat. 21, 147-152.

Shade, L. A., Oyama, F. J., Reinhert, A. and Miller, S. (1954). Serum iron and unsaturated iron binding capacity. Proc. Soc. exp. Biol. Med. 87, 443-448.

Tin Shwe, Sangaroon Suwansri and Mehler, H. (1972). Serum iron, total iron binding capacity and transferrin in leprosy. J. Burma Life Sci. 5, 331-333.

Turnbull, X. K. A. (1971). Microcytic hypochromic anaemias. Br. J. Hosp. Med. 6, 573-580. 